\title{
Nonmicrocephalic Infants with Congenital Zika Syndrome Suspected Only after Neuroimaging Evaluation Compared with Those with Microcephaly at Birth and Postnatally: How Large Is the Zika Virus "Iceberg"?
}

\author{
(D)M.F.V.V. Aragao, (D)A.C. Holanda, (D)A.M. Brainer-Lima, (D) N.C.L. Petribu, (D) M. Castillo, (D)V. van der Linden, (D) S.C. Serpa, \\ (D) A.G. Tenório, DP.T.C. Travassos, (DM.T. Cordeiro, (D) C. Sarteschi, (DM.M. Valenca, and (D)A. Costello
}

\begin{abstract}
BACKGROUND AND PURPOSE: Although microcephaly is the most prominent feature of congenital Zika syndrome, a spectrum with less severe cases is starting to be recognized. Our aim was to review neuroimaging of infants to detect cases without microcephaly and compare them with those with microcephaly.
\end{abstract}

MATERIALS AND METHODS: We retrospectively evaluated all neuroimaging (MR imaging/CT) of infants 1 year of age or younger. Patients with congenital Zika syndrome were divided into those with microcephaly at birth, postnatal microcephaly, and without microcephaly. Neuroimaging was compared among groups.

RESULTS: Among 77 infants, $24.6 \%$ had congenital Zika syndrome (11.7\% microcephaly at birth, 9.1\% postnatal microcephaly, $3.9 \%$ without microcephaly). The postnatal microcephaly and without microcephaly groups showed statistically similar imaging findings. The microcephaly at birth compared with the group without microcephaly showed statistically significant differences for the following: reduced brain volume, calcifications outside the cortico-subcortical junctions, corpus callosum abnormalities, moderate-to-severe ventriculomegaly, an enlarged extra-axial space, an enlarged cisterna magna (all absent in those without microcephaly), and polymicrogyria (the only malformation present without microcephaly). There was a trend toward pachygyria (absent in groups without microcephaly). The group with microcephaly at birth compared with the group with postnatal microcephaly showed significant differences for simplified gyral pattern, calcifications outside the cortico-subcortical junctions, corpus callosum abnormalities, moderate-to-severe ventriculomegaly, and an enlarged extra-axial space.

CONCLUSIONS: In microcephaly at birth, except for polymicrogyria, all patients showed abnormalities described in the literature. In postnatal microcephaly, the only abnormalities not seen were a simplified gyral pattern and calcifications outside the cortico-subcortical junction. Infants with normocephaly presented with asymmetric frontal polymicrogyria, calcifications in the cortico-subcortical junction, mild ventriculomegaly, and delayed myelination.

ABBREVIATIONS: CZS = congenital Zika syndrome; IgM = immunoglobulin M; PRNT = plaque reduction neutralization test; STORCH = syphilis, toxoplasmosis, rubella, cytomegalovirus, herpes simplex; ZIKV = Zika virus

$\mathbf{T}$ he Zika virus (ZIKV) is an arboviral disease with its main vector being Aedes aegypti. ${ }^{1}$ There are also reports of sexual transmission and viral detection in urine ${ }^{2}$ and tears. ${ }^{3}$ The first epidemic of ZIKV occurred in 2007 in the Yap Islands, Microne$\mathrm{sia}^{4}$; the second occurred in 2013, in French Polynesia ${ }^{5}$; and the

Received February 11, 2017; accepted after revision March 6.

From the Centro Diagnostico Multimagem (M.F.V.V.A.), Recife, Brazil; Federal University of Pernambuco (A.C.H.), Recife, Brazil; Pronto-Socorro Cardiológico de Pernambuco (Procape) (A.M.B.-L., M.M.V.), University of Pernambuco, Recife, Brazil; Barão de Lucena Hospital (N.C.L.P.), Recife, Brazil; Department of Radiology (M.C.), University of North Carolina, Chapel Hill, North Carolina; Association for Assistance of Disabled Children (V.v.d.L.), Recife, Brazil; Clínica de Apoio Ocupacional (S.C.S.), Jaboatão dos Guararapes, Brazil; Dom Malan Hospital (A.G.T.), Petrolina, Brazil; Laboratório Fernando Travassos (P.T.C.T.), Recife, Brazil; Centro de Pesquisas Aggeu Magalhães (M.T.C., C.S.), Fiocruz, Recife, Brazil; and Department of Maternal, Child, and Adolescent Health (A.C.), World Health Organization, Geneva, Switzerland.

third began in Bahia, Northeast Brazil, in March 2015. ${ }^{6}$ In August 2015, in Pernambuco, Northeast Brazil, a significant increase in the number of congenital microcephaly cases was reported to the health authorities. Currently, the relationship between the ZIKV and microcephaly is well-established. ${ }^{7}$

The most characteristic findings of congenital Zika syndrome (CZS) include microcephaly, arthrogryposis, and ophthalmologic and hearing abnormalities. ${ }^{8-12}$ The major neuroimaging abnormalities reported by initial case series ${ }^{8,13,14}$ were calcifications

\footnotetext{
Please address correspondence to Maria de Fatima Viana Vasco Aragão, MD, Rua Frei Matias Teves, 194 Ilha do Leite, Recife, PE, 50070-450 Brazil; e-mail: fatima.vascoaragao@gmail.com

- Indicates open access to non-subscribers at www.ajnr.org

三 Indicates article with supplemental on-line tables.

http://dx.doi.org/10.3174/ajnr.A5216
}

AJNR Am J Neuroradiol 38:1427-34 Jul 2017 www.ajnr.org 
in the cortico-subcortical white matter junction and malformations of cortical development, associated with other brain abnormalities. $^{8,13,14}$

These imaging features were reported on the basis of severe cases of microcephaly identified at birth. ${ }^{8,13-15}$ However, some of these patients ${ }^{8}$ did not have microcephaly at birth and were detected because in the beginning, microcephaly was defined as a head circumference of $\leq 33 \mathrm{~cm}$, a cutoff that decreased 2 times before the establishment of the current criteria based on the Intergrowth-21st. ${ }^{16}$ Therefore, there is probably a disease spectrum that has only recently been recognized, with some patients presenting with less severe brain damage and even without microcephaly.

We reviewed the brain CT and MR imaging scans of infants 1 year of age or younger, to find cases of CZS without microcephaly and to compare them with infants with microcephaly. We hypothesized that these mild cases of CZS without microcephaly, not suspected before neuroimaging evaluation, have a milder degree of brain damage.

\section{MATERIALS AND METHODS}

We retrospectively evaluated all brain MR imaging and CT scans of infants 1 year of age or younger from December 2015 to November 2016, in the Centro Diagnostico Multimagem Radiology Clinic. This study was approved by the research ethics committee.

The imaging reports were divided into 3 groups: 1) healthy patients, 2) nonspecific or not related to ZIKV, and 3) cases of neuroimaging abnormalities consistent with CZS. The last group was further subdivided into 3 groups: 1) infants with microcephaly at birth, 2) infants with microcephaly developed a few months after birth, and 3) infants without microcephaly. The classification of microcephaly was based on the Intergrowth-21st. ${ }^{16}$

Clinical information and CSF tests (syphilis, toxoplasmosis, rubella, cytomegalovirus, herpes simplex [STORCH] infections and ZIKV-specific immunoglobulin $\mathrm{M}[\operatorname{IgM}]$ ) were retrospectively reviewed in infants with microcephaly. In infants without microcephaly, a history of arboviral infection during pregnancy was queried for support of the MR imaging diagnosis, and laboratory investigation was performed.

Infants in this study were possible (suspected), probable, or confirmed cases of CZS. According to the Brazilian Ministry of Health, ${ }^{16}$ probable cases involve the following: 1) the mother's rash during pregnancy, 2) brain imaging suggestive of congenital infection, and 3) laboratory exclusion of STORCH infections in the mother and/or infant. Confirmed cases have, in addition, 4) laboratory confirmation of ZIKV infection in the mother and/or infant (eg, real-time reverse transcription polymerase chain reaction, ZIKV-specific IgM, plate reduction neutralization test [PRNT] for ZIKV in the CSF and/or serum). ${ }^{17}$ Suspected cases were classified as possible CZS when the above-mentioned items 1 or 4 were unknown or not present but the infant was born in an epidemic area and there was microcephaly and brain imaging suggestive of CZS. . $^{8,13,14}$

The group of infants with microcephaly at birth was compared with the group without microcephaly and with the group with postnatal microcephaly. We also joined groups and compared: 1) group with microcephaly at birth with group without microceph- aly at birth (postnatal microcephaly + without microcephaly); and 2) group with microcephaly (microcephaly at birth + postnatal microcephaly) with group without microcephaly. The images were analyzed by 2 experienced neuroradiologists (M.F.V.V.A. and A.M.B.-L.), with the final interpretation determined by a consensus. CT scans were subjectively reviewed for reduced brain volume; brain stem and cerebellar hypoplasia; malformations of cortical development; the presence and location of brain calcifications; corpus callosum abnormalities (classified as hypogenesis, when it was incompletely formed, or hypoplasia, when it was complete but reduced in thickness $)^{18}$; ventriculomegaly; an enlarged extra-axial CSF space; and an enlarged cisterna magna (largest diameters in 3 planes of $\geq 10 \mathrm{~mm}$ ). Symmetry of brain damage was determined by visual comparison between the cerebral hemispheres. All features identified on CT scans were also reviewed on brain MR imaging. In addition, we evaluated myelination (normal or delayed, according to milestones determined by a previous study) ${ }^{18}$ and types of malformations of cortical development classified as periventricular heterotopia, agyria, pachygyria, polymicrogyria, and simplified gyral pattern.

\section{Statistical Analysis}

For association, we used the Fisher exact test. A $P$ value $<.05$ was considered significant. The statistical analyses were performed by using SPSS software, Version 21.0 (IBM, Armonk, New York).

\section{RESULTS}

Regarding the neuroimaging diagnosis, among the 77 infants who underwent brain imaging scans (CT and/or MR imaging), 1) 18 (23.4\%) had normal examination findings; 2) 40 (52.0\%) had abnormalities considered nonspecific or not related to ZIKV infection; and 3) 19 (24.6\%) had neuroimaging abnormalities consistent with CZS. In the CZS group, 9 (11.7\%) infants had microcephaly at birth, $7(9.1 \%)$ had postnatal microcephaly, and 3 (3.9\%) did not have microcephaly. On-line Table 1 shows the individual clinical and laboratory data of the infants with CZS. The 3 infants without microcephaly were diagnosed as having probable CZS (On-line Table 2).

\section{Clinical Information}

The group with microcephaly at birth had more males (55.6\%), while the groups with postnatal microcephaly and without microcephaly had more females $(71.4 \%$ and $100.0 \%$, respectively), without statistical significance. Six of 9 mothers of infants with microcephaly at birth reported rashes (66.7\%), and 5 of 7 mothers of infants with postnatal microcephaly reported rashes (71.4\%). Other clinical information is shown in On-line Table 1. Ages at imaging evaluation were an average of $139.4 \pm 19.3$ days for microcephaly at birth; a median of 129 days (interquartile range, 88-328) for postnatal microcephaly; and 253,323, and 352 days for infants without microcephaly.

\section{Laboratory Status}

The laboratory tests were performed only once, shortly after birth for most infants (data from few of the infants could not be retrieved). For 2 infants with postnatal microcephaly and all with- 

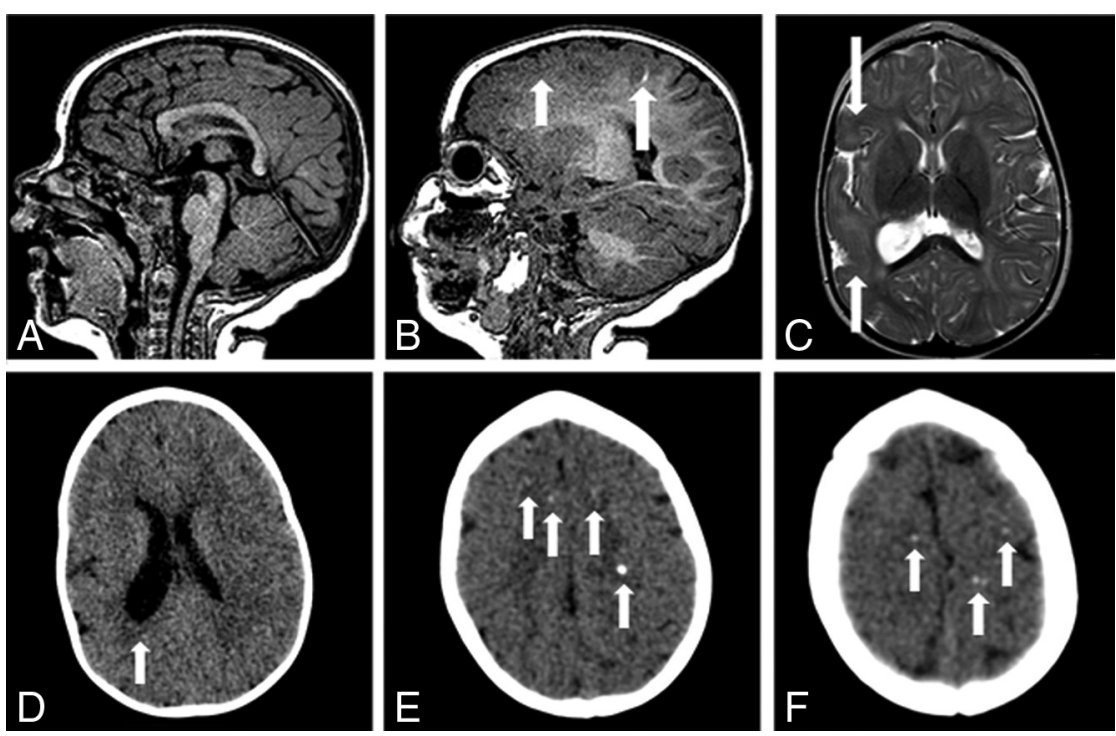

FIG 1. MR imaging and CT of an 8-month-old girl without microcephaly with probable congenital Zika syndrome. Sagittal T1-weighted image shows a normal corpus callosum and cisterna magna $(A)$ and a small hyperintense focus of dystrophic calcification in the junction between the cortical and subcortical white matter (long white arrow) and left frontal polymicrogyria (short white arrow) (B). Axial T2-weighted image $(C)$ shows right polymicrogyria (white arrows) and mildly decreased right hemisphere volume. CT scans show asymmetric hemispheres, with an enlarged right lateral ventricle (white arrow) (D) and small punctate foci, representing calcifications, at the cortico-subcortical white matter junction bilaterally in the frontal lobes (white arrows) ( $E$ and $F$ ).
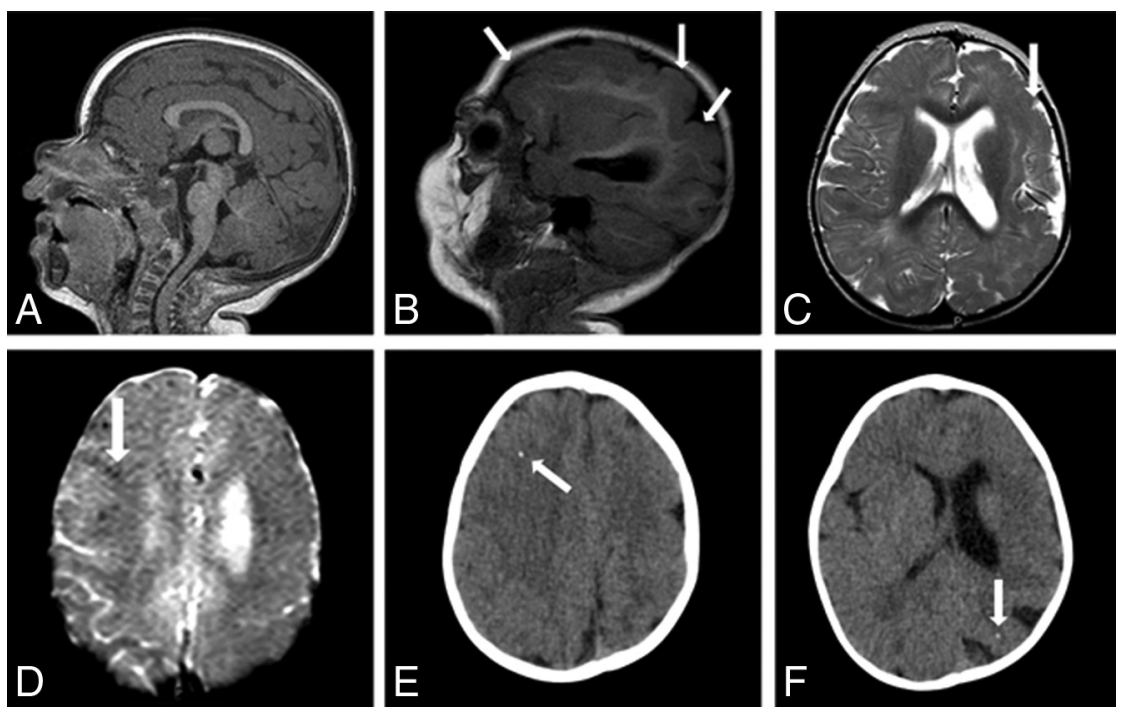

FIG 2. MR imaging and CT of an 11-month-old girl without microcephaly with probable congenital Zika syndrome. Sagittal T1-weighted image shows a normal corpus callosum and cisterna magna $(A)$ and left polymicrogyria (white arrows) (B). Axial T2-weighted image $(C)$ shows left polymicrogyria (white arrow) and mild left ventriculomegaly. A gradient-echo image $(D)$ shows very few small and subtle punctate foci, representing calcifications, at the cortico-subcortical white matter junction (white arrow). Axial CT scans ( $E$ and $F$ ) show right frontal and left parietal punctate foci (white arrows).

out microcephaly (infants 15-19), tests were performed shortly before or after imaging evaluation; therefore, close to 1 year of age.

Findings of tests for STORCH and dengue in the CSF were negative in 18 infants (data not available for Infant 10) (On-line Table 1). In the microcephaly at birth group, $7 / 8$ infants tested for Zika-specific IgM in the CSF had positive findings and just 1/8 had negative results (On-line Table 1). In the postnatal microcephaly group, 2/4 infants tested for Zika-specific IgM in the CSF had positive findings. The other $2 / 4$ who had negative results also had negative PRNT test findings in the CSF, but they were tested only several months after birth (On-line Table 1). All 3 infants with normal head circumferences had negative ZIKV-specific IgM and PRNT test findings in the CSF. However, the serologic PRNT test had positive results in both tested mothers of 2 infants without microcephaly (On-line Table 1).

\section{Neuroimaging Features}

Imaging abnormalities in the group without microcephaly (Figs 1-3) were not significantly different from those in the group with postnatal microcephaly (Fig 4).

The group without microcephaly was significantly different from the one with microcephaly at birth (Fig 5) for the following: reduced brain volume, calcifications outside the cortico-subcortical white matter junction, corpus callosum abnormalities, moderate-tosevere ventriculomegaly, an enlarged extra-axial CSF space, and an enlarged cisterna magna (Table). All these features were found only in microcephaly at birth. In addition, polymicrogyria was not present in the microcephaly at birth group, a finding that was significantly different from that in the group without microcephaly.

The group with microcephaly at birth was significantly different from the one with postnatal microcephaly in the following features: a simplified gyral pattern, calcifications outside the cortico-subcortical white matter junction, corpus callosum abnormalities, moderate-to-severe ventriculomegaly, and an enlarged extra-axial CSF space (Table). All these features were found more frequently in the microcephaly at birth group. In addition, polymicrogyria was not present in the microcephaly at birth group, with statistical differences from the group without microcephaly.

When the groups with and without microcephaly at birth were compared, significant differences were found for brain stem hypoplasia, simplified gyral pattern, calcifications outside the cortico-subcortical white matter junction, corpus callosum abnormalities, moderate-to-severe ventriculomegaly, an enlarged extra-axial CSF space, and an enlarged cisterna magna (Table). All these features were found more frequently or exclusively in the microcephaly at birth group. Polymicrogyria was not present in the microcephaly at birth 

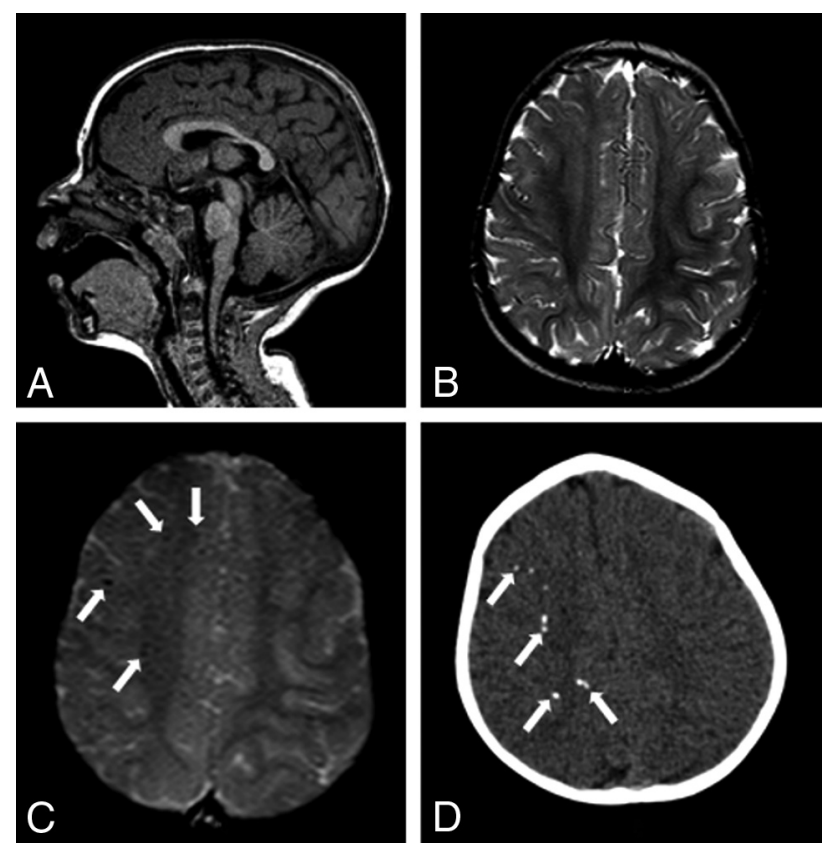

FIG 3. MR imaging and CT of an 11-month-old girl without microcephaly with probable congenital Zika syndrome. Sagittal $\Pi$-weighted image shows a normal corpus callosum and cisterna magna (A). Axial T2weighted image $(B)$ shows a normal cortex. A gradient-echo image $(C)$ shows small and subtle punctate foci, representing calcifications, at the cortico-subcortical white matter junction (white arrows). Axial CT scan (D) shows punctate foci in the right hemisphere (white arrows).
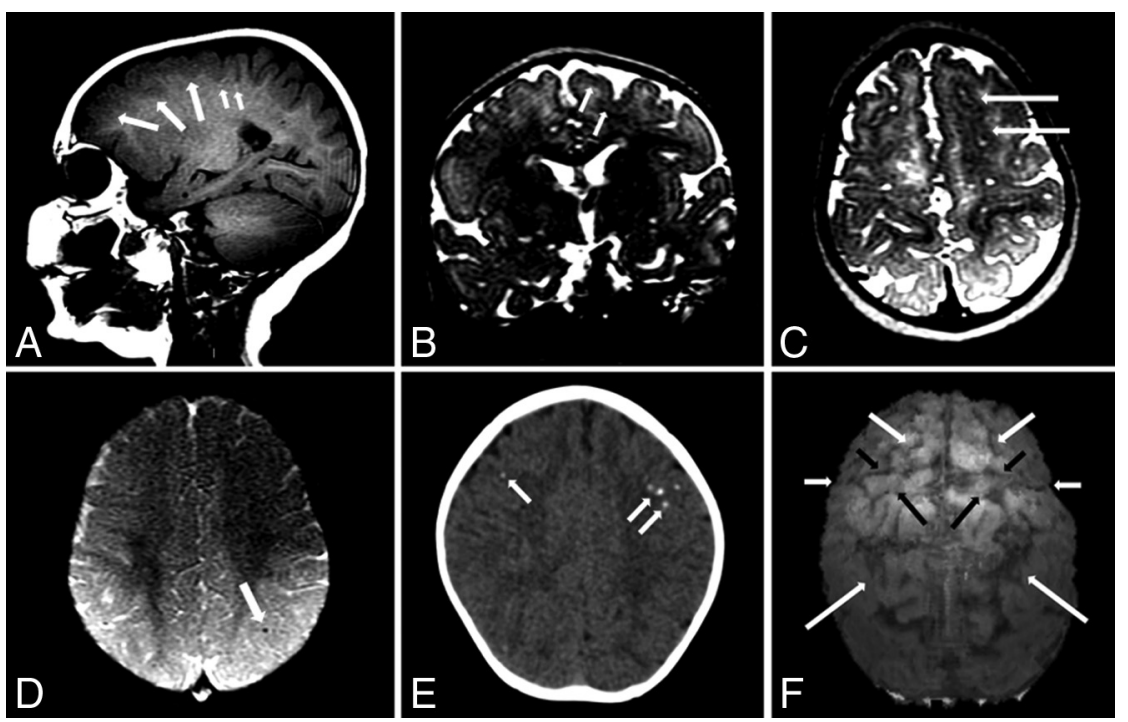

FIG 4. MR imaging and CT of a 10-month-old girl with microcephaly developed postnatally with possible congenital Zika syndrome. Sagittal T1-weighted image shows frontal polymicrogyria (medium white arrows) and very subtle hyperintense punctate foci, representing calcifications at the cortico-subcortical white matter junction (small white arrows). Coronal and axial T2weighted images ( $B$ and $C$, respectively) show the thick and irregular cortex at the superior frontal sulcus (white arrows). Axial SWI (D) shows a small punctate focus, representing calcification at the cortico-subcortical white matter junction (white arrow). Axial CT scan (E) shows punctate foci in both frontal lobes. An echo-spoiled gradient-echo volumetric (3D reconstruction) image $(F)$ shows malformation and prominence of brain high frontoparietal convexity gyri and sulci, predominantly at the left hemisphere: The superior frontal sulcus (medium white arrows) is wellidentified bilaterally, as well as precentral (short black arrows), central (short white arrows), and intraparietal (long white arrows) sulci. The left hemisphere is more reduced than the right hemisphere; the left precentral gyrus (large black arrows) seems to be the most reduced in volume. These findings are located probably where the polymicrogyria is most severe, according to the T2-weighted images ( $B$ and $C$ ). These findings are better seen in a 3D reconstruction $(F)$ than in sectional images $(A-C)$, despite the presence of movement artifacts in the former. group. Cerebellar hypoplasia was present in only 3 infants with

icrocephaly at birth, with a statistical trend. extra-axial CSF space, and an enlarged cisterna magn (Table). All of these features were found only in infants with

\section{DISCUSSION}

Among the 77 infants studied, 16 (20.8\%) with microcephaly the healthy population. However, this percentage could be either higher, because these cases are only starting to be detected, ico Multimagem radiologic center was higher because it

\section{Neuroimaging Features of the Congenital Zika Syndrome} Spectrum

Infants born with microcephaly presented with the highest number of abnormalities and more severe malformations, followed by those with postnatal microcephaly and then by the infants without microcephaly. However, significant differences were not observed between the last 2 groups.

Reduction in brain volume is present in all infants with microcephaly, while brain volume is preserved in infants without microcephaly with significant differences. Therefore, the normal head size in the normocephalic group is not due to hydrocephalus ${ }^{14}$ but due to less severe brain damage. Volumetric MR imaging studies could be useful for better characterizing the reduction of brain volume. This information could help in understanding the physiopathology of the disease, in the differential diagnosis, and in the prognosis and follow-up of these infants.

Moderate-to-severe ventriculomegaly was significantly more frequent in the group with microcephaly at birth compared with the other 2 groups, both separately and jointly. Ventricular enlargement is directly related to the reduction of brain volume, which is more severe in the group with microcephaly at birth and not due to hydrocephalus.

The frequency of enlarged extra-axial CSF spaces increases with the severity of the CZS spectrum. It was present in all infants with microcephaly at birth and 

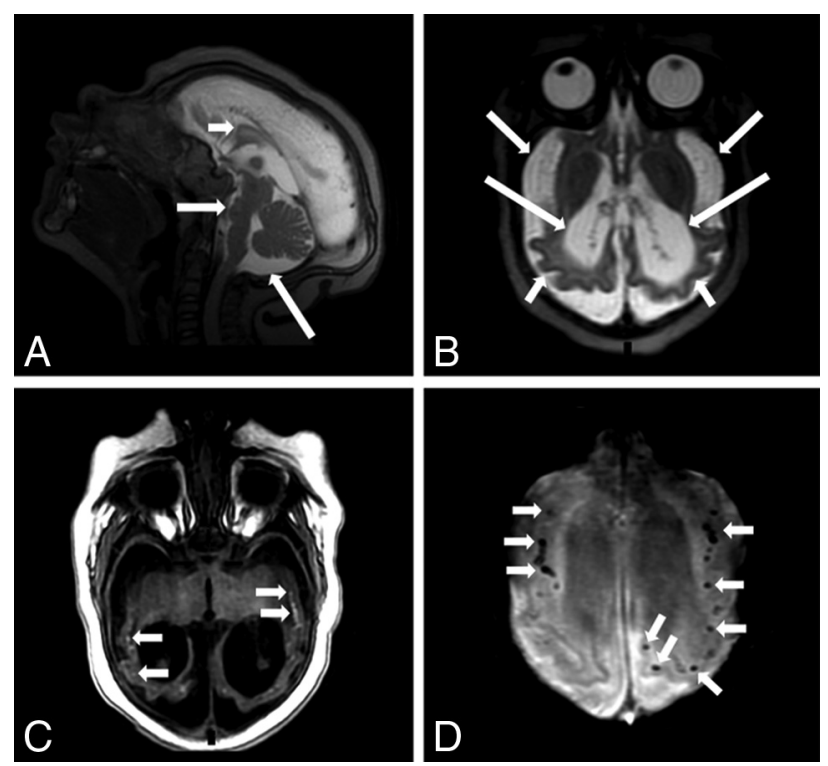

FIG 5. MR imaging of a 4-month-old boy with microcephaly with confirmed congenital Zika syndrome. Sagittal T2-weighted image $(A)$ shows a hypogenetic corpus callosum (short white arrow), pons hypoplasia (medium white arrow), and an enlarged cisterna magna (long white arrow). An axial T2-weighted image (B) shows a diffuse simplified gyral pattern (note the thin cortex) (short white arrows), an enlarged extra-axial CSF space (medium white arrows), and severe ventriculomegaly (long white arrows). An axial T1-weighted image (C) shows hyperintense punctate foci, representing calcifications, at the cortico-subcortical white matter junction (small white arrows). Axial SWI (D) shows several punctate foci at the cortico-subcortical white matter junction (short white arrows). absent in infants without microcephaly, with significant differences being directly related to reduced brain volume. Enlarged extra-axial space could also be the result of impaired CSF reabsorption.

The frequency of an enlarged cisterna magna also increases with the severity of the CZS spectrum. It was present in all infants with microcephaly at birth and absent in infants without microcephaly. Significant differences were found in most comparative analyses (microcephaly at birth versus postnatal microcephaly showed a statistical trend). An enlarged cisterna magna is not related to cerebellar hypoplasia because this was an infrequent finding.

Brain stem hypoplasia is mainly present in the most severe cases with microcephaly at birth. This group was significantly different from the combined group without microcephaly at birth. The decreased brain stem volume, especially of the pons, could represent more severe damage in microcephaly at birth, being possibly explained by a decreased number of descending fibers, supported by anatomic-pathologic studies. ${ }^{20}$ Another hypothesis is direct viral action in brain stem nuclei. DTI studies could help in clarifying ZIKV damage not only in the brain stem but also to fibers in other sites.

A simplified gyral pattern was characteristic of microcephaly at birth, being exclusively found in this group and significantly different compared with the combined group without microcephaly at birth. This finding suggests that this type of malformation, resulting from diminished cell proliferation or white matter development in utero, ${ }^{18}$ is associated with more severe damage. In a simplified gyral pattern, few neurons are produced or too many undergo apoptosis. ${ }^{18}$

Comparison of brain MRI findings between microcephalic and normocephalic infants with congenital Zika syndrome

\begin{tabular}{|c|c|c|c|c|c|c|c|}
\hline \multirow[b]{2}{*}{ MRI Findings } & \multicolumn{2}{|c|}{ Microcephaly } & \multirow{2}{*}{$\begin{array}{c}\text { Without } \\
\text { Microcephaly } \\
(n=3)\end{array}$} & \multirow[b]{2}{*}{$P_{1}$} & \multirow[b]{2}{*}{$P_{2}$} & \multirow[b]{2}{*}{$P_{3}$} & \multirow[b]{2}{*}{$P_{4}$} \\
\hline & $\begin{array}{c}\text { At Birth } \\
(n=9)\end{array}$ & $\begin{array}{c}\text { Postnatally } \\
(n=7)\end{array}$ & & & & & \\
\hline Reduced brain volume & $8(88.9 \%)$ & $5(71.4 \%)$ & $0(0.0 \%)$ & $.018^{\mathrm{a}}$ & .550 & .141 & $.021^{\mathrm{a}}$ \\
\hline Brain stem hypoplasia & $6(66.7 \%)$ & $1(14.3 \%)$ & $0(0.0 \%)$ & .182 & .060 & $.020^{\mathrm{a}}$ & .263 \\
\hline Cerebellum hypoplasia & $3(33.3 \%)$ & $0(0.0 \%)$ & $0(0.0 \%)$ & .509 & .213 & .087 & 1.000 \\
\hline \multicolumn{8}{|l|}{ Malformations of cortical development ${ }^{b}$} \\
\hline Simplified gyral pattern & $6(66.7 \%)$ & $0(0.0 \%)$ & $0(0.0 \%)$ & .182 & $.028^{\mathrm{a}}$ & $.009^{a}$ & .515 \\
\hline Pachygyria & $6(66.7 \%)$ & $4(66.7 \%)$ & $0(0.0 \%)$ & .182 & 1.000 & .637 & .069 \\
\hline Polymicrogyria & $0(0.0 \%)$ & $4(66.7 \%)$ & $2(66.7 \%)$ & $.045^{\mathrm{a}}$ & $.011^{\mathrm{a}}$ & $.009^{a}$ & .245 \\
\hline Normal cortex & $1(11.1 \%)$ & $0(0.0 \%)$ & $1(33.3 \%)$ & .455 & 1.000 & 1.000 & .314 \\
\hline \multicolumn{8}{|l|}{ Brain calcifications } \\
\hline Cortico-subcortical white matter junction & $8(88.9 \%)$ & 7 (100.0\%) & $3(100.0 \%)$ & 1.000 & 1.000 & .474 & 1.000 \\
\hline Calcifications at other sites & $7(77.8 \%)$ & $0(0.0 \%)$ & $0(0.0 \%)$ & $.046^{\mathrm{a}}$ & $.003^{\mathrm{a}}$ & $.001^{\mathrm{a}}$ & .263 \\
\hline Basal ganglia & $4(44.4 \%)$ & $0(0.0 \%)$ & $0(0.0 \%)$ & .491 & .103 & .082 & 1.000 \\
\hline Thalamus & $3(33.3 \%)$ & $0(0.0 \%)$ & $0(0.0 \%)$ & .509 & .229 & .206 & 1.000 \\
\hline Periventricular & $3(33.3 \%)$ & $0(0.0 \%)$ & $0(0.0 \%)$ & .509 & .229 & .206 & 1.000 \\
\hline Brain stem & $4(44.4 \%)$ & $0(0.0 \%)$ & $0(0.0 \%)$ & .491 & .103 & .082 & 1.000 \\
\hline Cerebellum & $2(22.2 \%)$ & $0(0.0 \%)$ & $0(0.0 \%)$ & 1.000 & .475 & .471 & 1.000 \\
\hline Corpus callosum abnormalities & $9(100.0 \%)$ & $2(28.6 \%)$ & $0(0.0 \%)$ & $.005^{\mathrm{a}}$ & $.005^{\mathrm{a}}$ & $.001^{\mathrm{a}}$ & .058 \\
\hline Moderate-to-severe ventriculomegaly & $8(88.9 \%)$ & $2(28.6 \%)$ & $0(0.0 \%)$ & $.018^{\mathrm{a}}$ & $.035^{\mathrm{a}}$ & $.005^{\mathrm{a}}$ & .087 \\
\hline Enlarged extra-axial CSF space & $9(100.0 \%)$ & $3(42.9 \%)$ & $0(0.0 \%)$ & $.005^{\mathrm{a}}$ & $.019^{\mathrm{a}}$ & $.003^{\mathrm{a}}$ & $.036^{\mathrm{a}}$ \\
\hline Enlarged cisterna magna & $9(100.0 \%)$ & $4(57.1 \%)$ & $0(0.0 \%)$ & $.005^{\mathrm{a}}$ & .063 & $.008^{\mathrm{a}}$ & $.021^{\mathrm{a}}$ \\
\hline Delayed myelination ${ }^{\mathrm{b}}$ & $7(77.8 \%)$ & $2(33.3 \%)$ & $2(66.7 \%)$ & 1.000 & .136 & .335 & 1.000 \\
\hline Symmetry of abnormalities & $8(88.9 \%)$ & $3(42.9 \%)$ & $1(33.3 \%)$ & .127 & .106 & .057 & .523 \\
\hline
\end{tabular}

Note: $-P$ indicates Fisher exact test; $P_{7}$, microcephaly at birth vs without microcephaly; $P_{2}$, microcephaly at birth vs microcephaly postnatally; $P_{3}$, microcephaly at birth vs without microcephaly at birth (microcephaly postnatally + without microcephaly); $P_{4}$, microcephaly (microcephaly at birth + microcephaly postnatally) vs without microcephaly. Microcephaly postnatally vs without microcephaly yielded no statistically significant results.

a Statistically significant results.

${ }^{\mathrm{b}}$ Not assessed in Infant 14 (without MRI). For myelination assessment, infant 15 was considered born at term. 
Pachygyria was present exclusively in infants in the combined group with microcephaly (at birth and postnatally). Despite these findings, only a trend was found when these groups were compared, possibly due to the small number of infants without microcephaly. Pachygyria, as a simplified gyral pattern, probably represents more severe brain damage, being associated with other abnormalities. When present in the same infant with microcephaly at birth, pachygyria was located in the anterior brain (a thick cortex mainly in the frontal lobes), and a simplified gyral pattern comprised mostly the posterior parts of the brain (parietal and occipital lobes).

Polymicrogyria was the only malformation of cortical development found in infants without microcephaly, and it was absent in infants with microcephaly at birth. This finding reinforces polymicrogyria being probably a less severe lesion, with consequent preservation of brain volume and the corpus callosum. In postnatal microcephaly, when polymicrogyria and pachygyria were found in the same infant, the former was located in the anterior brain (mainly in the frontal lobes), while the latter was located in the posterior parts of the brain (mainly in the parietal and occipital lobes).

Brain calcifications in the cortex and subcortical white matter were found in almost all CZS cases. Calcifications located at other sites (basal ganglia, thalamus, periventricular, brain stem, and cerebellum) were found only in microcephaly at birth, with significant differences compared with the other 2 groups, both separately and combined. When they are distributed in several structures of the brain, they suggest more severe damage and, consequently, probably more severe brain volume reduction. Even though CT has higher sensitivity to intracranial calcifications, MR imaging is also able to detect them. Calcifications appear as hyperintense foci on T1-weighted images, especially when the brain is not completely myelinated, and as hypointense on SWI, which is the best sequence to demonstrate them. ${ }^{8,21}$

The frequency of corpus callosum abnormalities (hypogenesis and hypoplasia) is directly related to CZS severity. Corpus callosum abnormalities were absent in infants without microcephaly and very frequent in the infants with microcephaly at birth. Significant differences were found in most comparative analyses (patients with microcephaly versus those without microcephaly showed a statistical trend). Hypogenesis or hypoplasia of the corpus callosum with microcephaly at birth could be related to more severe parenchymal damage and cortical malformation, with a consequent decreased number of fibers crossing the cerebral hemispheres.

Lesions were more symmetric in infants with microcephaly at birth compared with infants without it, with a strong trend. Possibly this difference is because microcephaly at birth is related to more diffuse and severe lesions. In regards of the physiopathology, it is knwon that the ZIKV attacks neural progenitor cells and mature neural cells, causing downregulation of genes involved in cell cycle pathways, dysregulation of cell proliferation, and upregulation of genes involved in apoptotic pathways resulting in cell death. ${ }^{22}$ However, some authors suggest that vascular phenomena could also be involved in the pathophysiology. ${ }^{14,23}$ Heterogeneous material in the confluence of the sinuses, probably corresponding to thrombus, has already been described. ${ }^{14,23}$ As the use of contrast is usually not recommended in infants, the frequency of cerebral venous thrombosis is unknown. Nevertheless, we hypothesize that, in CZS, there may exist an important damage to the cerebral vascular system, especially in the venous component, leading to cerebral venous thrombosis and cerebral venous hypertension during intrauterus development and continuing after birth. This could explain the cortico-subcortical calcifications, the development of hydrocephalus in some of the infants, ${ }^{24}$ and changes in brain lesions, ${ }^{23,25}$ similarly to what happens in cerebral venous hypertension of the physiopathology of vein of Galen aneurysmal malformations. ${ }^{26}$ The confirmation of this vascular hypothesis could lead to research for identifying treatment options during pregnancy and after birth, improving the prognosis of the infants and the evolution of the disease. The identification of changes in parechyma thickness, ventricle size and calcifications ${ }^{23,25}$ indicates that these infants should be followed not only clinically, but also by laboratory tests (possibility of persistent viral activity) and by neuroimaging, especially if there are signs of disease progression.

In summary, the most severe group, with microcephaly at birth, presented with all the major abnormalities already described in the literature (reduced brain volume, brain stem hypoplasia, cerebellar hypoplasia, a simplified gyral pattern, pachygyria, calcifications at the cortico-subcortical white matter junction and at other sites, corpus callosum abnormalities, moderate-to-severe ventriculomegaly, an enlarged extra-axial CSF space, an enlarged cisterna magna, and delayed myelination), except for polymicrogyria.

In the intermediate group with postnatal microcephaly, the only abnormalities absent were simplified gyral pattern and calcifications outside the cortico-subcortical junction, compared with infants with microcephaly at birth. On the other hand, polymicrogyria was found in infants with postnatal microcephaly and without microcephaly but not in infants with microcephaly at birth.

In the less severe extreme, without microcephaly, there was only asymmetric polymicrogyria, mainly in the frontal lobes, calcifications restricted to the cortical/white matter junctions, mild ventricular enlargement, and delayed myelination.

\section{Congenital Zika Syndrome without Microcephaly}

The clinical signs of brain impairment in the 3 children without microcephaly were noticed by their mothers several months after birth. They were nonspecific, and the diagnosis could have been missed without accurate MR imaging interpretation. The 3 mothers remembered a rash during pregnancy, but 2 of them recognized it only when they were asked after the MR imaging report. Because about $75 \%$ of infected patients may be asymptomatic, ${ }^{27}$ imaging becomes important in showing the "tip of the iceberg."

Although other infections were excluded in all infants without microcephaly, Zika-specific tests did not confirm the ZIKV infection in these infants. However, these Zika-specific tests are not completely trustworthy, because they are not validated yet. ${ }^{28}$ For IgM in the CSF, this negative result was expected due to the long period since the infection occurred because the children are currently close to 1 year of age. For PRNT (IgM and immunoglobin $\mathrm{G})$, the negative results in these infants are difficult to understand because the 2 mothers tested for PRNT (cases 18 and 19) had 
positive results and all 3 had rashes during pregnancy, supporting ZIKV infection. Thus, although these 3 infants with negative specific tests are probable CZS, ${ }^{17}$ we suggest they be considered to have confirmed CZS.

The negative PRNT results and lower severity and number of brain lesions in infants without microcephaly need future experimental studies to be clarified. More severe ZIKV damage could be related to earlier vertical transmission during pregnancy, higher viral load, and a crossed immunologic response, among other hypotheses. Environmental factors could also be involved because the most severe cases are located in Northeast Brazil. ${ }^{14}$

Maternal infection during pregnancy was more frequent in the first trimester for microcephaly and in the second trimester for normocephaly, though without significant differences. One limitation is that the maternal ZIKV infection was based exclusively on recall of the mothers' rashes during pregnancy. Mothers may not remember the exact month in which they had the rash, and this drawback limits our interpretations. In addition, the literature has shown that the interval between maternal infection and sonographic evidence of fetal abnormalities can range from 2 to 27 weeks. $^{20}$

These are the first published cases in which CZS was not suspected by neuropediatricians because the patients had normal head circumferences and nonspecific neurologic signs and the diagnosis was only raised after MR imaging evaluation. They raise an important epidemiologic issue: How many children have less severe CZS brain damage and are being missed?

The radiologist's role is even more important in CZS without microcephaly: to recognize subtle suggestive findings and to indicate the probable diagnosis to the neuropediatrician. This early diagnosis allows rehabilitation to start as soon as possible, improving the development of these infants. Clinical and radiologic follow-up of the brains will be important to understand the pathophysiology of this new disease.

Our study has limitations, especially due to the small sample and retrospective method. Studies with a larger number of infants are needed to confirm the results and to reinforce information that was identified here. Nevertheless, the study was able to characterize brain abnormalities in 3 degrees of the CZS spectrum with statistically significant differences.

\section{CONCLUSIONS}

Infants without clinical suspicion of CZS (without microcephaly) can be first identified with neuroimaging studies. These cases represented $3.9 \%$ of the infants in our survey.

The spectrum of CZS comprises 3 different degrees of severity, with many statistically significant differences among the groups: 1) CZS with microcephaly at birth, which presented, except for polymicrogyria, with all abnormalities previously described in the literature; 2) CZS with postnatal microcephaly, in which, in relation to the group with microcephaly at birth, only a simplified gyral pattern and calcifications outside the cortical/white matter junctions were absent, but the infants presented with polymicrogyria; and 3) CZS without microcephaly, which presented with only asymmetric polymicrogyria mainly in the frontal lobes, calcifications restricted to the cortical/white matter junctions, mild ventricular enlargement, and delayed myelination.

\section{ACKNOWLEDGMENTS}

We thank Antônio Monteiro, Cristovam A de Lira Terceiro, Lucas Coelho Belfort Lustosa, Edineide Cristina Lopes, and Islane Martins for their inestimable help.

\section{REFERENCES}

1. Dick GW, Kitchen SF, Haddow AJ. Zika virus, I: isolations and serological specificity. Trans $R$ Soc Trop Med Hyg 1952;46:509-20 CrossRef Medline

2. Gourinat AC, O'Connor O, Calvez E, et al. Detection of Zika virus in urine. Emerg Infect Dis 2015;21:84-86 CrossRef Medline

3. Miner JJ, Sene A, Richner JM, et al. Zika virus infection in mice causes panuveitis with shedding of virus in tears. Cell Rep 2016;16: 3208-18 CrossRef Medline

4. Duffy MR, Chen TH, Hancock WT, et al. Zika virus outbreak on Yap Island, Federated States of Micronesia. N Engl J Med 2009;360: 2536-43 CrossRef Medline

5. Cauchemez S, Besnard M, Bompard P, et al. Association between Zika virus and microcephaly in French Polynesia, 2013-15: a retrospective study. Lancet 2016;387:2125-32 CrossRef

6. Campos GS, Bandeira AC, Sardi SI. Zika virus outbreak, Bahia, Brazil. Emerg Infect Dis 2015;21:1885-86 CrossRef Medline

7. Rasmussen SA, Jamieson DJ, Honein MA, et al. Zika virus and birth defects: reviewing the evidence for causality. N Engl J Med 2016;374: 1981-87 CrossRef Medline

8. de Fatima Vasco Aragao M, van der Linden V, Brainer-Lima AM, et al. Clinical features and neuroimaging (CT and MRI) findings in presumed Zika virus related congenital infection and microcephaly: retrospective case series study. BMJ 2016;353: i1901 CrossRef Medline

9. Leal MC, Muniz LF, Caldas Neto SD, et al. Sensorineural hearing loss in a case of congenital Zika virus. Braz J Otorhinolaryngol 2016 Jun 30. [Epub ahead of print] CrossRef Medline

10. Ventura CV, Maia M, Ventura BV, et al. Ophthalmological findings in infants with microcephaly and presumable intra-uterus Zika virus infection. Arq Bras Oftalmol 2016;79:1-3 CrossRef Medline

11. van der Linden V, Filho EL, Lins OG, et al. Congenital Zika syndrome with arthrogryposis: retrospective case series study. BMJ 2016;354:i3899 CrossRef Medline

12. Schuler-Faccini L, Ribeiro EM, Feitosa IM, et al; Brazilian Medical Genetics Society-Zika Embryopathy Task Force. Possible association between Zika virus infection and microcephaly: Brazil, 2015. MMWR Morb Mortal Wkly Rep 2016;65:59-62 CrossRef Medline

13. Hazin AN, Poretti A, Turchi Martelli CM, et al. Computed tomographic findings in microcephaly associated with Zika virus. $N$ Engl J Med 2016;374:2193-95 CrossRef Medline

14. Soares de Oliveira-Szejnfeld P, Levine D, Melo AS, et al. Congenital brain abnormalities and Zika virus: what the radiologist can expect to see prenatally and postnatally. Radiology 2016;281:203-18 CrossRef Medline

15. Poretti A, Huisman TA. Neuroimaging findings in congenital Zika syndrome. AJNR Am J Neuroradiol 2016 Aug 4. [Epub ahead of print] CrossRef Medline

16. Ministério da Saúde (Brazil). Protocolo de vigilância e resposta à ocorrência de microcefalia e/ou alterações do sistema nervoso central (SNC). 2016. http://combateaedes.saude.gov.br/images/salade-situacao/Microcefalia-Protocolo-de-vigilancia-e-resposta-10mar 2016-18h.pdf. Accessed April 15, 2017

17. Ministério da Saúde (Brazil). Orientações integradas de vigilância e atenção à saúde no âmbito da Emergência de Saúde Pública de Importância Nacional. 2016. http://portalsaude.saude.gov.br/images/ pdf/2016/dezembro/12/orientacoes-integradas-vigilancia-atencao. pdf. Accessed December 20, 2016

18. Adachi Y, Poduri A, Kawaguch A, et al. Congenital microcephaly with a simplified gyral pattern: associated findings and their significance. AJNR Am J Neuroradiol 2011;32:1123-29 CrossRef Medline

19. Agência Estadual de Planejamento e Pesquisas de Pernambuco. 
Natalidade: Anuário Estatístico de Pernambuco. 2015. http:// www.anuario.pe.gov.br/demografia/natalidade. Accessed April 15, 2017

20. Melo AS, Aguiar RS, Amorim MM, et al. Congenital Zika virus infection: beyond neonatal microcephaly. JAMA Neurol 2016;73: 1407-16 CrossRef Medline

21. Livingston JH, Stivaros S, Warren D, et al. Intracranial calcification in childhood: a review of aetiologies and recognizable phenotypes. Dev Med Child Neurol 2014;56:612-26 CrossRef Medline

22. Tang H, Hammack C, Ogden SC, et al. Zika virus infects human cortical neural progenitors and attenuates their growth. Cell Stem Cell 2016;18:587-90

23. Aragão MF, Brainer-Lima AM, Holanda AC, Petribu NC de L. Neuroimaging findings of congenital Zika syndrome. In: Aragão MF, ed. Zika in Focus. Springer International Publishing; 2017:63-92

24. van der Linden V, Filho EL, van der Linden A. Congenital Zika syndrome: clinical aspects. In: Aragão MF, ed. Zika in Focus. Springer International Publishing; 2017:33-45
25. Petribu NC, Aragão MF. Cases: Spectrum of Computed Tomography in Congenital Zika Syndrome. In: Aragão MF, ed. Zika in Focus. Springer International Publishing; 2017:107-24

26. Diebler C, Dulac O, Renier D, et al. Aneurysms of the vein of Galen in infants aged 2 to 15 months: diagnosis and natural evolution. $\mathrm{Neu}$ roradiology 1981;21:185-97

27. Besnard M, Lastere S, Teissier A, et al. Evidence of perinatal transmission of Zika virus, French Polynesia, December 2013 and February 2014. Euro Surveill 2014;19 Medline

28. de Araújo TV, Rodrigues LC, de Alencar Ximenes RA, et al; investigators from the Microcephaly Epidemic Research Group, Brazilian Ministry of Health, Pan American Health Organization, Instituto de Medicina Integral Professor Fernando Figueira, State Health Department of Pernambuco. Association between Zika virus infection and microcephaly in Brazil, January to May, 2016: preliminary report of a case-control study. Lancet Infect Dis 2016;16:1356-63 CrossRef Medline 